\title{
Should ambulant patients be directed to reception or triage first?
}

\author{
S Goodacre, F Morris, B Tesfayohannes, G Sutton
}

\begin{abstract}
Objectives-Developments in triage have led to patients being directed to triage rather than reception upon arrival in A\&E. This study aimed to discover if attending triage or reception first, was preferable in terms of timeliness, clarity and safety.

Methods-The study compared two consecutive four week periods during which patients were directed to attend triage first and then reception first. Observers recorded their actions on arrival. Questionnaires recorded the patients' perceptions of each strategy. High priority triage categories were audited during the reception first phase.

Results-1850 patients were observed in the triage first phase with a mean door to triage time of $\mathbf{1 0 . 6}$ minutes, triage to reception 5.3 minutes and door to reception 15.8 minutes. A total of 1522 patients were observed in the reception first phase with a mean door to reception time of 0.5 minutes, reception to triage $\mathbf{1 2 . 4}$ minutes and door to triage of 12.9 minutes. Patients were more likely to present to the appropriate place during the reception first phase $(88 \%$ versus $34 \%)$ and reported better understanding of instructions. No case given triage category one or two suffered an adverse outcome resulting from delay associated with attending reception first.

Conclusion-Directing patients to attend reception first is timely and less confusing. It is safe provided booking in does not delay assessment by more than 15 minutes.

(Emerg Med f 2001;18:441-443)
\end{abstract}

Keywords: triage; patient satisfaction

Traditionally the first point of contact for ambulant patients attending the accident and emergency (A\&E) department was the reception desk. In recent years there has been increasing recognition of the importance of nurse triage, ${ }^{1}$ with the development of standardised triage categories, guidelines ${ }^{2}$ and national targets ${ }^{3}$ for times to triage. The need to prompt early assessment by a triage nurse has led many departments to direct ambulant patients to the triage area rather than reception.

This approach has been adopted by the Northern General Hospital (NGH) A\&E department for several years. Patients arriving at the $\mathrm{A} \& \mathrm{E}$ department entrance are guided by signs to the triage assessment area where they are assessed before booking at reception. However, staff noted that many patients seemed to disregard the signs and present themselves to reception first. It was therefore suggested that the arrangement be changed to prompt patients to attend reception first. This suggestion raised concerns that patients attending with time critical complaints might be inadvertently delayed at reception.

To resolve the issue we undertook a comparative study of the two different protocols-patients to attend triage first or to attend reception first. We aimed to answer the following questions - which protocol resulted in most rapid assessment overall, which protocol did patients follow appropriately, which protocol did patients prefer, and could receptionists recognise patients ultimately triaged to emergency categories.

\section{Methods}

The NGH A\&E department is the only department serving the city of Sheffield, although ambulant patients may attend a minor injuries unit at the Royal Hallamshire Hospital. A total of 75000 patients attend the NHG A\&E each year. Ambulant patients attend by a separate entrance from the ambulance borne. Both reception and the triage area are adjacent to the entrance. The triage room is separated from the waiting room by a door that directly faces the entrance. This door is kept open except when patients are being examined. Reception opens onto the waiting room via desks that face perpendicular to the entrance. Signs immediately in front of the entrance direct patients to the appropriate area. Patients are triaged into standard, nationally recognised categories, ${ }^{2}$ according to local guidelines.

The study took place over two consecutive four week periods in October and November 1999. During the first phase patients were, as normal, directed to attend triage first. During the second phase patients were directed to attend reception first. For matched periods during each phase observers recorded the movements of all patients attending $\mathrm{A} \& \mathrm{E}$ via the ambulant entrance. The following time periods were observed-10 00 am to $200 \mathrm{pm}$ (Monday to Friday), $500 \mathrm{pm}$ to $900 \mathrm{pm}$ (Monday to Friday), $1200 \mathrm{pm}$ to $800 \mathrm{pm}$ (Saturday and Sunday) and $1000 \mathrm{pm}$ to 200 am (Friday, Saturday and Sunday). The observers recorded the time each patient arrived at the entrance, their actions upon arrival, the time they were seen at reception and the time they were seen at triage.

During the same two phases a convenience sample of questionnaires were distributed to patients attending $A \& E$ via the ambulant entrance. The questions used are described in the results section. 
Finally, during the reception first phase of the study, reception staff had to complete a mandatory computer coding upon booking in each patient. Upon entering patient details they were asked to code whether they felt the patient needed immediate attention by the triage nurse or not. Over the same period all ambulant patients given triage categories one or two (attention required immediately or within 10 minutes) were identified.

Two senior members of medical staff (BT and SG) independently reviewed the notes of all category one or two patients who had not been identified by reception staff. Each decided whether: (1) the delay associated with attendance at reception first could have adversely affected the patients' outcome, and (2) a theoretical delay associated with reception of at least 15 minutes could have adversely affected the patients' outcome. Decisions were compared and discrepancies resolved by a third party (FM).

Routine data collected by the NGH Patient Administration System computer were used to determine the total number of attendances during each study period and the characteristics of those who did not arrive by ambulance.

Statistical analysis was carried out using SPSS for Windows (Version 6.1.3). Categorical variables were compared using the Pearson $\chi^{2}$ test.

\section{Results}

The triage first phase of the study ran from the 4 October to the 31 October 1999 and the reception first phase from the 1 November to the 28 November. During the triage first phase there were a total of 7467 attendances, of whom $5427(72.7 \%)$ did not attend by ambulance. During the reception first phase there were a total of 7619 attendances, of whom $5678(74.5 \%)$ did not attend by ambulance. Complete routine hospital data relating to age, sex, triage categorisation and disposal were available for $4756(87.6 \%)$ and $4922(86.7 \%)$ of each group, respectively. These details are outlined in table 1 .

The actions of 3372 patients were recorded by observers during the study period. Some 1850 during the triage first phase, 1522 during the reception first phase. This represents 34\% and $27 \%$ respectively of all ambulant attendances during the two phases of the study. Absences among observers reduced the total time observed. During the triage first phase 66 of 68 observation periods were covered, whereas during the reception first phase only 59 of 68 were covered. No particular time period seemed to be disproportionately affected by absent observers.

Table 1 Ambulant AEE attendances during the two study periods

\begin{tabular}{lll}
\hline & Triage first & Reception first \\
\hline Total & 4756 & 4922 \\
Admitted & $431(9 \%)$ & $396(8 \%)$ \\
Cases in triage categories 1 or 2 & $29(0.61 \%)$ & $27(0.55 \%)$ \\
Mean age & 38 & 37 \\
Men & $2715(57.1 \%)$ & $2880(58.5 \%)$ \\
\hline
\end{tabular}

Table 2 Responses to "Was it clear to you where to go and what to do when you arrived at $A \mathcal{E} E$ ?"

\begin{tabular}{lll}
\hline & Triage first & Reception first \\
\hline Yes & $190(85.2 \%)$ & $288(92 \%)$ \\
No & $33(14.8 \%)$ & $25(8 \%)$ \\
\hline
\end{tabular}

Response rate 536 of $536=100 \%$. p $=0.012$.

Table 3 Responses to "After seeing the assessing nurse, did you feel that you had enough information about how long could expect to wait?"

\begin{tabular}{llll}
\hline & All & Triage first & Reception first \\
\hline Yes & $482(92 \%)$ & $183(85.5 \%)$ & $299(96.5 \%)$ \\
No & $42(8 \%)$ & $31(14.5 \%)$ & $11(3.5 \%)$ \\
\hline
\end{tabular}

Response rate 524 of $536=97.8 \%$. $p=0.00001$.

Table 4 Responses to "After seeing the assessing nurse, did you feel that you had enough information about what priority your injury had been given?"

\begin{tabular}{llll}
\hline & All & Triage first & Reception first \\
\hline Yes & $291(67.1 \%)$ & $84(48.3 \%)$ & $207(79.6 \%)$ \\
No & $143(32.9 \%)$ & $90(51.7 \%)$ & $53(20.4 \%)$ \\
\hline
\end{tabular}

Response rate 434 of $536=81 \%$. p $<0.00001$.

Table 5 Responses to "Who would you like to see first?"

\begin{tabular}{llll}
\hline & All & Triage first & Reception first \\
\hline Assessing nurse & $253(50.2 \%)$ & $142(67.6 \%)$ & $111(37.8 \%)$ \\
Receptionist & $251(49.8 \%)$ & $68(32.4 \%)$ & $183(62.2 \%)$ \\
\hline
\end{tabular}

Response rate 504 of $536=94 \%$. p $<0.00001$.

The mean door to triage times were-10.6 minutes for the triage first group and 12.9 minutes for the reception first group $(\mathrm{p}<0.0001$, Mann-Whitney U test). The mean door to reception times were- 15.8 minutes for the triage first group and 0.5 minutes for the reception first group ( $<<0.0001$, MannWhitney $U$ test). The mean reception to triage time was 12.4 minutes (reception first group only) and the mean triage to reception time was 5.3 minutes (triage first group only).

During the triage first phase, $58.2 \%$ of patients initially presented to reception, while $34.0 \%$ of the patients presented, as instructed, to triage. During the reception first phase $88.3 \%$ presented, as instructed, to reception first $(\mathrm{p}<0.00001)$.

A total of 536 questionnaires were returned-313 from those attending reception first, 223 from those attending triage first. Responses are outlined in tables 2 to 5 .

During the reception first phase of the study reception staff coded 113 patients $(2 \%)$ as requiring immediate attention. During the same period 27 ambulant patients were given emergency triage categories (that is, categories 1 or 2). One patient had suffered a cardiac arrest in the department and another was brought in with a respiratory arrest. Both bypassed triage and reception and were therefore excluded. Of the remaining 25 patients, six $(24 \%)$ were identified by reception staff as requiring immediate attention.

Review of those not identified by reception staff found no case in which the delay caused by attending reception could have significantly affected outcome. This was principally because delays associated with reception were minimal (less than five minutes) in all time critical cases 
and eventual outcomes were good. However, six cases were identified in which outcome could have been adversely affected if booking in at reception had delayed presentation to triage by more than 15 minutes. These werethree cases of myocardial infarction (two requiring thrombolysis), two cases of significant vaginal bleeding and one allergic reaction.

\section{Discussion}

The direction of ambulant patients attending $A \& E$ may seem to be a trivial issue for clinicians. For patients, however, it represents their first contact with the department and occurs at a time when they may be anxious or distressed. Unexplained waits and a confusing environment will exacerbate such feelings and may impair subsequent relationships with clinical staff. Early impressions therefore have a powerful effect upon patients' perceptions of the quality of care they receive, while patient satisfaction is inversely associated with waiting time. ${ }^{4}$

By attending reception first, patients had contact with a member of staff sooner $(0.5 v$ 10.6 minutes) and completed booking in and assessment quicker (12.9 $v 15.8$ minutes). This strategy only delayed presentation at triage by approximately two minutes (10.6 v 12.9). These results probably reflect the fact that reception staff are permanently available to attend to patients while the triage nurse may be delayed by prolonged assessments or occupied by other duties.

The majority of patients initially went to reception regardless of the strategy in place. Questionnaire responses indicated that directions were more clear during the reception first phase of the trial. Most patients (92\%) were satisfied with the information they received about waiting times, although significantly more $(96.5 \%$ \% 85.5\%) were satisfied during the reception first phase. Approximately two thirds of patients were satisfied with the information they received regarding the priority their injury had been given. Again, satisfaction was higher during the reception first phase of the trial $(79.6 \% v 48.3 \%)$. Overall patients were equally divided in their preference to see either the triage nurse or the receptionist first. During each phase of the trial the majority favoured the protocol they were allocated to.

These findings may either reflect improved giving of information during the reception first phase or improved recollection of information. Triage nurses reported feeling under less time pressure when they were no longer the first point of contact in the department and may therefore have been better able to give clear information. Alternatively, patients may fail to recollect information given to them at triage after they have completed booking in at reception.

Reception staff were unable to accurately identify patients requiring immediate assessment. However, the time delay caused by attending reception first was so short compared with time delays for triage, that we could not identify any negative impact upon patient care. This would not hold true if delays incurred by booking in at reception exceeded 15 minutes. It should be noted that reception staff had received no training in recognition of immediately time critical illness. It is possible that a brief intervention or guideline might improve their performance.

This study has potential limitations that should be appreciated. Firstly, the questionnaire was administered in an opportunistic manner and it is not known how many were given out and the proportion returned. It is conceivable therefore that those returned may be a biased sample. However, as patients were not aware of the study hypothesis, it is not clear whether such bias would favour those with positive or negative views. There is no strong reason to suspect that bias is responsible for our results favouring a reception first strategy.

It is possible that absence among observers during the second phase could have lead to bias in the time periods studied. However, the times of absences were distributed throughout the week and it is unlikely that this potential bias could have been responsible for the magnitude of change detected in our results.

Both triage and reception staff were aware of the study and could have changed their normal behaviour in response to being observed (the Hawthorne effect). This might have improved the efficiency with which both groups of staff worked, but it is not clear how it would effect the overall results.

The time period and number of cases used to assess the safety of the reception first approach is somewhat small and it is possible that a longer period of assessment would have revealed cases of adverse outcome related to delay at reception. As a result of this study we will be changing to the reception first strategy. The method used to evaluate safety described in this study will be continued, with regular re-evaluations, to ensure that this approach is safe over a longer time period.

Finally, it may be argued that our findings are attributable to limited availability of the triage nurse and could be reversed by providing more triage support. However, the level of training and experience required by a triage nurse dictates that these personnel are likely to be in more frequent demand than reception staff. It would be useful to repeat this study elsewhere to determine whether these problems are unique to the NGH, or are an inevitable consequence of the triage role.

In conclusion, this study shows that at the $\mathrm{NGH}$ a policy of prompting patients to attend reception first is timely and less confusing, both objectively to an external observer and subjectively to the patient. The safety of this strategy depends upon delays at reception being no more than 15 minutes.

Funding: none.

Conflicts of interest: none.

1 Williams DG. Sorting out triage. Nursing Times 1992;88:34-

2 Manchester Triage Group. Emergency triage. London: BMJ Publishing, 1997.

3 Department of Health. The patients charter. London: HMSO, 1991 .

4 Bailey A, Hallam K, Hurst K. Nursing practice Bailey A, Hallam K, Hurst K. Nursing practice
supplement-triage on trial. Nursing Times 1987;83:65-6. 